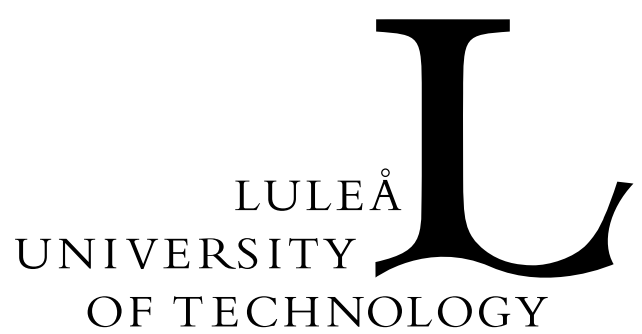

Division of Signal Processing

Visiting address: Universitetsområdet, Porsön, Luleå Postal address: SE-971 87, Luleå, Sweden

Telephone: +46920910 00. Fax: +4692072043

URL: http://www.sm.luth.se/csee/sp/

\title{
Synchronization of a TDMA-OFDM \\ Frequency Hopping System
}

\author{
Jan-Jaap van de Beek, Per Ola Börjesson, \\ Marie-Laure Boucheret, Daniel Landström, \\ Julia Martinez Arenas, Per Ödling, Sarah Kate Wilson, \\ Christer Östberg and Mattias Wahlqvist
}

In Proceedings of Vehicular Technology Conference (VTC '98), Vol 2, pp. 12901294, Ottawa, Canada, May 1998.

(C) 1998 IEEE. Personal use of this material is permitted. However, permission to reprint/republish this material for advertising or promotional purposes or for creating new collective works for resale or redistribution to servers or lists, or to reuse any copyrighted component of this work in other works must be obtained from the IEEE. 


\title{
Synchronization of a TDMA-OFDM frequency hopping system
}

\author{
Jan-Jaap van de Beek ${ }^{1} \quad$ Per Ola Börjesson ${ }^{1} \quad$ Marie-Laure Boucheret $^{2}$ \\ Daniel Landström ${ }^{1} \quad$ Julia Martinez Arenas ${ }^{2} \quad$ Per Ödling ${ }^{1}$ \\ Sarah Kate Wilson ${ }^{1}$ Christer Östberg ${ }^{3} \quad$ Mattias Wahlqvist ${ }^{4}$ \\ ${ }^{1}$ Luleå University, Div. of Signal Processing, SE-971 87 Luleå, Sweden \\ ${ }^{2}$ Ecole National Superieure de Telecommunications, Site de Toulouse, BP4004 CEDEX, Toulouse, France \\ ${ }^{3}$ Ericsson Mobile Communications AB, SE-221 83 Lund, Sweden. \\ 4 Telia Research AB, SE-977 75 Luleå, Sweden.
}

\begin{abstract}
In this paper we present a Time Division Multiple Access - Orthogonal Frequency Division Multiplexing (TDMA-OFDM)-based radio interface proposal for the Universal Mobile Telecommunication System (UMTS). We focus on synchronization and present an implementable algorithm for the tracking of the mobiles' time and frequency offsets at the base station in the uplink. This algorithm is based on recently developed estimation methods that use redundancy introduced by the cyclic prefix. A modified Maximum Likelihood (ML) estimator for the Additative White Gaussian Noise (AWGN) channel is used to meet both implementational requirements and performance requirements for frequency-selective fading multiuser environments. Simulation results for a typical UMTS mobile channel environment show that the uncoded symbol error rate of a coherently modulated system using our synchronization scheme is virtually indistinguishable from that of a system with no time and frequency offset.
\end{abstract}

\section{INTRODUCTION}

This paper presents a multiuser Time Division Multiple Access - Orthogonal Frequency Division Multiplexing (TDMA-OFDM) system with a scheme for synchronization in the uplink. This work is part of the evaluation process of different access proposals for the future Universal Mobile Telecommunication System (UMTS) which is currently being standardized within the European Telecommunications Standards Institute (ETSI) [1]. A transmission scheme and multiple access scheme that are believed to fulfill these requirements are proposed in [2]. The described system uses the OFDM transmission technology [3]. How to synchronize different users in the multiuser uplink of OFDM-based systems is a frequently asked question that has to date had no clear answer. Proper synchronization contributes to the orthogonality of the users, which is essential for reliable transmission.
The optimal joint Maximum Likelihood (ML) estimator of time and frequency offsets for the AWGN channel, has been presented in [4] and [5]. This estimator is, however, explicitly evaluated for a broadcast or downlink scenario. In this paper, we show that statistical redundancy in the received signal can be successfully exploited in the uplink operating in a dispersive, fading environment.

We present an implementable estimator structure based on results in [4] for the case of a narrowband uplink signal using coherent detection. Because the signal is narrowband, the offset estimation is not as accurate as in a wideband OFDM systems. However, if the time offset error is within part of the cyclic prefix, the channel estimation algorithm, required for coherent detection, compensates for the error. We evaluate the performance of the UMTS scenario employing this estimator in the base station in terms of uncoded symbol error rate.

\section{The Multiuser Scenario}

We consider a system where the available spectrum in a cell is accessed by a large number of subcarriers [2]. This system allows for high and variable bit rates, as well as dynamic channel allocation in order to handle traffic load variations between cells. Data symbols are efficiently modulated on these carriers by means of a Fast Fourier Transform (FFT) [3], both in the uplink and the downlink.

UMTS has been assigned frequencies in the $2.2 \mathrm{GHz}$ band. The uplink and downlink use different frequency bands each $4.27 \mathrm{MHz}$ wide, containing 1024 subcarriers. The intercarrier spacing is thus $4.17 \mathrm{kHz}$ and the symbol length is $240 \mu \mathrm{sec}$. Each symbol is cyclically extended by $48 \mu$ sec (205 samples). The $48 \mu$ sec is chosen to be longer than the channel impulse in the system and to be compatible with the Global System for Mobile communication (GSM) time slot structure [6]. The system uses Quadrature Phase Shift Keying (QPSK) modulation. In OFDM systems employing a cyclic prefix the frequency-selective time-varying channel distortion appears as a multiplicative distortion of the transmitted data symbols [3]. In order to 


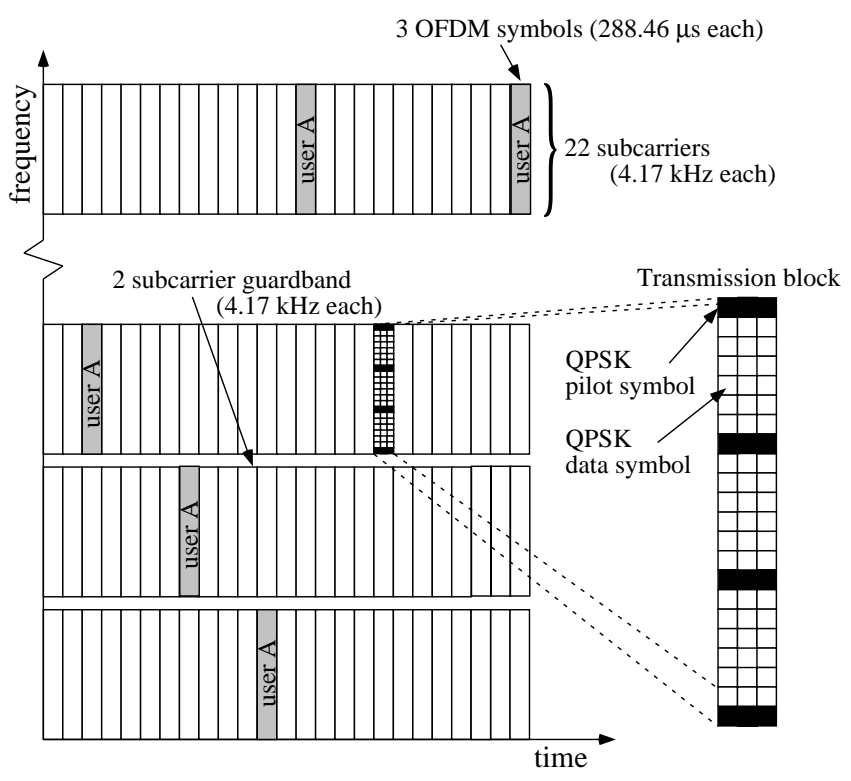

Figure 1 - Time-frequency grid for the UMTS scenario. The users are assigned transmission blocks consisting of 22 adjacent subcarriers and 3 adjacent OFDM symbols. Each transmission block contains pilot symbols supporting the channel estimation.

coherently detect the QPSK-data symbols, the proposed system estimates and compensates for these attenuations [2]. For this purpose pilot symbols are transmitted. The use of coherent detection and pilot symbols for channel estimation supports a future expansion of the signal alphabet to multiamplitude constellations. The pilot symbols for channel estimation require about $20 \%$ of the transmission capacity. Table 1 summarizes the basic system parameters.

\begin{tabular}{|l|l|}
\hline Center frequency & $2.2 \mathrm{GHz}$ \\
\hline UL / DL bandwidth & $4.27 \mathrm{MHz} / 4.27 \mathrm{MHz}$ \\
\hline Intercarrier spacing & $4.17 \mathrm{kHz}$ \\
\hline Symbol length & $240 \mu \mathrm{sec}(1024$ samples $)$ \\
\hline Cyclic prefix & $48 \mu \mathrm{sec}(205$ samples $)$ \\
\hline Transmission block & 22 subcarriers $\times 3 \mathrm{symb}$. \\
\hline Data rate data & multiples of $5.7 \mathrm{kbits} / \mathrm{sec}$. \\
\hline $\begin{array}{l}\text { Minimum uncoded } \\
\text { rate }\end{array}$ & $5 \mathrm{Mbits} /$ second \\
\hline $\begin{array}{l}\text { Maximum uncoded data } \\
\text { rate }\end{array}$ & $5.1 \mathrm{~kb}$. \\
\hline
\end{tabular}

Table 1. Characteristics of the UMTS proposal

Figure 1 illustrates the multiple access scheme we will adopt, which separates users both in time and in frequency by means of an access grid [2]. As a minimal access entity, every user is assigned 22 adjacent subcarriers that occupy 3 adjacent OFDM symbols. Each transmission block has 1 empty guardband carrier on either side. We will refer to such an entity as a transmission block. This concept will enable us to separate multiple user signals at the base station. For a typical low-rate circuit switched service, such as a speech service, 3 such transmission blocks are allocated and transmitted every $18.47 \mathrm{msec}$ according to a predetermined frequency-hopping pattern. For higher data rates, users request more transmission blocks. The time and frequency diversity in the system is recovered by the use of a convolutional code. The proposed convolutional code is a rate $1 / 2$, constraint length 7 , code [2]. The evaluation of this code is beyond the scope of this paper.

\section{Synchronization in the Multiuser Scenario}

To synchronize in the uplink of our UMTS scenario, we propose a scheme with three parts: a bank of bandpass filters, a time and frequency offset estimator, and a downlink control channel.

First, the base station employs a filterbank which separates the different transmission blocks' signals, so that each transmission block's offsets can be estimated. Second, the base station estimates these offsets using the redundancy introduced by the cyclic prefix. Third, to preserve system orthogonality, all users need to adjust to the base station's reference clock and oscillator. As opposed to downlink synchronization, the clock- and oscillator adjustments must be made at the mobile's transmitter. For this reason we propose a downlink control channel on which the estimates are fed-back from the base station to the mobiles.

\section{A. Synchronization Requirements}

Accurate demodulation and detection of an OFDM signal requires subcarrier orthogonality. Impairment of the carrier oscillator, the sample clock or the symbol clock may affect the orthogonality of the system. In this paper we focus on two of these impairments, the symbol timing offset $\theta$ and the carrier frequency offset $\varepsilon$. For this purpose we adopt the model

$$
r(k)=s(k-\theta) e^{j 2 \pi \varepsilon k / N}+n(k),
$$

where $s(k)$ and $r(k)$ are the sampled baseband transmitted and received OFDM signal, and $n(k)$ is additive white Gaussian noise. We now briefly address impairments of the carrier oscillator (in particular the frequency offset $\varepsilon$ ), the sample clock and the symbol clock (in particular the timing offset $\theta$ ).

First, the frequency and phase of the transmitter carrier oscillator may differ from those of the reference oscillator. A carrier phase offset results in a phase offset of the data symbols on each subcarrier, but does not cause any Intersymbol Interference (ISI) or Intercarrier Interference (ICI) [7]. Also, in a system with coherently modulated subcarriers, carrier phase synchronization is performed by the channel equalizer. A carrier frequency offset $\varepsilon$, however, causes a loss of orthogonality between the tones resulting in ICI [8]. Since the system bandwidth is small compared to the carrier frequency, we assume that each subcarrier is equally affected. The analysis of multiuser OFDM systems in $[9][8]$ shows that a frequency accuracy of $1 \%-2 \%$ 
of the inter-carrier spacing is necessary to obtain a signalto-interference ratio of $30 \mathrm{~dB}$ or higher. In our system this translates to a frequency offset of about $50 \mathrm{~Hz}$ (equivalent to $1.3 \%$ of the intercarrier spacing).

Secondly, the frequency and phase of the sample clock in the transmitter may differ from those of the reference receiver clock. For instance, in our scenario a sample clock specification of $10 \mathrm{ppm}$ may yield a clock drift of about 43 samples per second. In this paper we ignore the effects of sample clock offsets. These effects are investigated in [10].

Finally, since the demodulator FFT processes blocks of $N$ samples the transmitted signals must be time-aligned in order to avoid ISI. The symbol clock frequency is derived from the sample clock frequency which we assume is known. In this paper we focus on the unknown time offset $\theta$ of the symbol clock: that is, which block of $N$ samples the receiver must process in the FFT. The channel estimator cannot distinguish between phase shifts introduced by the radio channel and those introduced by symbol timing delays [11]. Our system uses a cyclic prefix of $48 \mu \mathrm{sec}$. This prefix accommodates both the delay spread of the channel and an offset of the symbol clock phase. As long as the accumulated effect of a symbol clock phase offset and a far echo in the channel impulse response does not exceed $48 \mu \mathrm{sec}$, the system performance is equal to a system with a known symbol clock. In Section IV we will see that in the UMTS scenario employing our synchronization scheme the symbol timing offset rarely exceeds $14 \mu \mathrm{sec}$ (60 samples). Therefore, the system accomodates channel impulse responses with echos up to $34 \mu$ sec. Note that this figure exceeds that of the GSM standard, which accommodates channel echos up to $16 \mu$ sec [6]

\section{B. Base station receiver structure}

In Figure 2 the receiver structure of the base station is shown. It consists of two parallel parts. In one part, the cyclic prefix is removed and the data are demodulated by means of an FFT. The demodulated symbols are corrected with respect to the multiplicative channel distortion and the result is fed into the detector. The other part serves to generate time and frequency offset estimates. The received sampled baseband signal is fed into a bank of filters, each selecting the particular frequencies of one transmission block. For each resulting signal, the time and frequency offsets are estimated. The filterbank can be efficiently realized using a polyphase and FFT implementation [12].

\section{Time and frequency offset estimation}

We propose a modification to a time and frequency offset estimator that works without the aid of pilot symbols and is independent of the modulation of the carriers [4]. The estimator exploits the statistical redundancy introduced by the cyclic prefix to estimate the information about the offsets from the different transmission blocks. This estimator has been presented in [12]. Here we briefly review

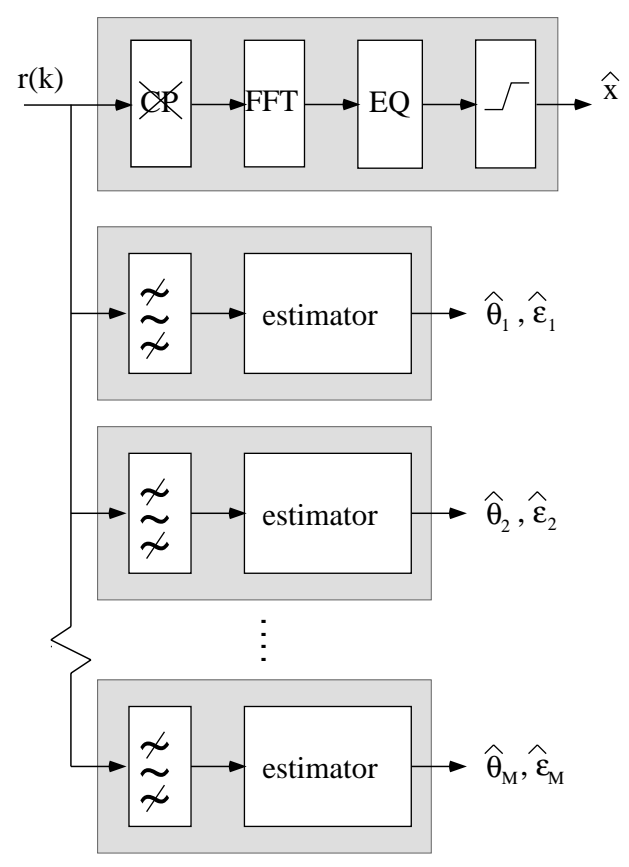

Figure 2 - The structure of the receiver at the base station. One path serves the detection of the data, while other paths estimate the time and frequency offsets for each user.

the basis of the proposed estimator.

We assume that $M$ mobile users transmit data to the base station. We consider the sampled received OFDM signal, composed of $22 \mathrm{M}$ subcarriers, and we associate with each transmission block $m$ a time offset $\theta_{m}$ relative to the receiver symbol clock and a frequency offset $\varepsilon_{m}$ relative to the receiver demodulation frequency. Since the bank of bandpass filters in the receiver isolates each transmission block's redundancy, we model the output of filter $m$ as

$$
r_{m}(k)=s_{m}\left(k-\theta_{m}\right) e^{j 2 \pi \varepsilon_{m} k / N}+n(k), m=1, \ldots, M,
$$

where $s_{m}(k)$ is the signal transmitted on transmission block $m$ and $n(k)$ is additive complex white Gaussian receiver noise. The signal $r_{m}(k)$ can be used to estimate $\theta_{m}$ and $\varepsilon_{m}$.

In a mobile multiuser scenario it is fair to assume that consecutive symbols do not differ much in their arrival time and frequency offset. For that reason, we can use redundancy in consecutive symbols to improve the estimates of $\theta_{m}$ and $\varepsilon_{m}$. The proposed estimator is based on a weighted average of the joint maximum likelihood function of $\theta_{m}$ and $\varepsilon_{m}$, and is implemented by means of an Infinite Impulse Response (IIR) filter to reduce memory requirements. The exponential weighting is suitable in environments where $\theta_{m}$ and $\varepsilon_{m}$ vary slowly and the delay associated with the averaging does not affect the performance.

The channel estimator cannot distinguish between phase rotations introduced by the time-varying dispersive channel and phase rotations introduced by a carrier frequency offset or a small symbol timing offset [11]. Therefore, the 
channel equalizer not only compensates for the attenuations of the time-varying dispersive channel, but also corrects for a part of the time offset. The performance of the proposed synchronization scheme is thus evaluated in a system with channel estimation and equalization. We use a generically designed pilot based Mimimum Mean Square Error (MMSE) channel estimator addressed in [13].

\section{Control Channel}

Since all users must arrive at the base station with a certain synchrony in order to maintain orthogonality between the subcarriers, all users adapt to the base station receiver clock and oscillator. For this reason the base station entertains a control channel on the downlink for every connected user. Control information, based on the offset estimates, is transmitted back to the user. This is similar to the GSM standard [6]. Apart from time and frequency offsets, other control parameters are transmitted on the control channel. These parameters include which transmission blocks to use for the uplink transmission, which frequency hopping pattern to use, and also which transmission power to apply. Successful tracking synchronization of the user thus relies on the control channel.

\section{System PERFormanCE}

In this section we evaluate the performance of the time and frequency offset estimator as well as the uplink performance of the UMTS proposal. In simulations we use the ETSI Vehicular A channel model [1] and the weight in the averaging of the likelihood function is 0.75 .

We illustrate the tracking ability of the time and frequency offset estimator in Figures 3 and 4 . To make the estimator's tracking ability more clear, we model the time offset with a sinusoid with 30 samples amplitude and one period every 96 OFDM symbols for one specific user. The frequency offset also follows a sinusoid with the same period as the time offset and an amplitude of $5 \%$ of the intercarrier spacing. Note that such a model for the drift in time and frequency offsets is unrealistic in our UMTS scenario, because of the large and rapid variations. The model however, serves the purpose of showing the tracking ability.

Now we evaluate the uplink performance of the UMTS proposal employing the channel estimator and the proposed time and frequency offset estimator. In the simulations (Table 2 and Figure 5) we assume a linear symbol clock drift of $10 \mathrm{ppm}$ and a constant carrier frequency offset. Table 2 shows the proposed estimator's standard deviations and the percentage of estimates being outside the specified intervals. For the time-offset estimate these percentages together with the length of the channel dispersion show how often ISI will occur and when the requirements, as stated in section III, cannot be fulfilled.

Figure 5 shows the performance of the proposed TDMAOFDM system in terms of uncoded symbol error rate (SER). Three cases are simulated. First, a system with

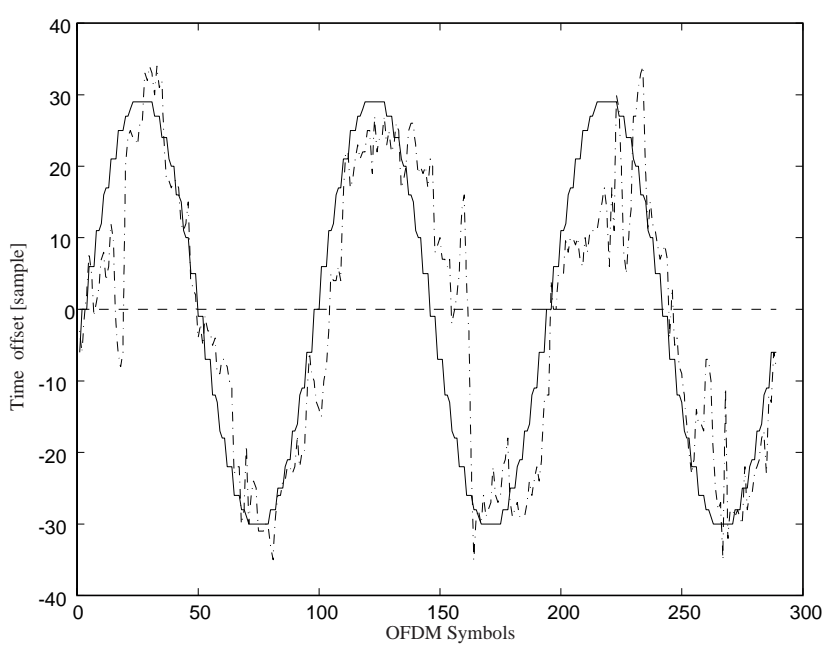

Figure 3 - Timing offsets versus time. The true timing offset (solid) and the estimated time offset (dashed).

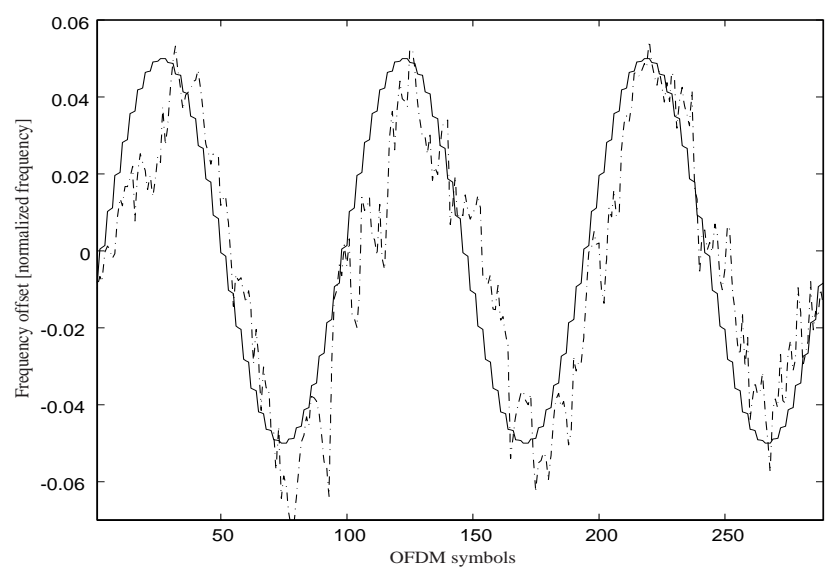

Figure 4 - Frequency offsets versus time. The true frequency offset (solid) and the estimated frequency offset (dashed).

perfect synchronization and ideal channel knowledge in the receiver equalizer. Second, a system without time and frequency offsets but employing the channel estimator and equalizer. Finally, a system employing the channel estimator but also using fed-back estimates of the time and frequency offsets in the transmitter. Estimates are fedback every 48:th transmission block (10 times a second). The performance of a system employing our synchronization scheme is not visibly decreased compared to the system performance with no time and frequency offsets, since the channel estimator compensates for small time offsets.

\section{SUMMARY AND CONCLUSIONS}

In this paper we review a TDMA-OFDM based multiuser system for mobile communications and we focus on the synchronization in the uplink. Time and frequency offsets are estimated at the base station. Control informa- 


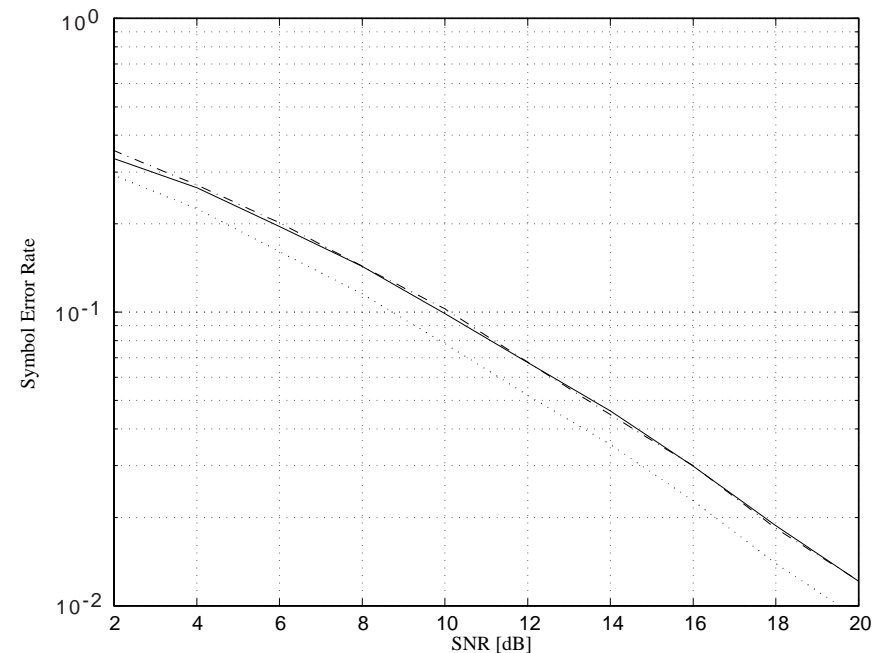

Figure 5 - The symbol error rate of the UMTS scenario with QPSK modulated subcarriers and channel estimation/equalization. System performance without synchronization errors and known channel (dotted); system performance without synchronization errors but with channel estimation (solid); system performance for the proposed synchronization scheme (dash-dotted).

tion based on these estimates is transmitted on a downlink control channel to the user, who adjusts its symbol clock and its local oscillator accordingly. The estimation is done by exploiting the statistical redundancy introduced by the cyclic prefix.

We give parameter choices, simulation results, and implementation suggestions for a particular proposal for an UMTS radio interface and show that the synchronization requirements are satisfied. Simulations show that the performance of a coherently modulated system employing our synchronization scheme is virtually indistinguishable decreased compared to the performance of a system with perfect time and frequency offset estimation.

\section{REFERENCES}

[1] ETSI-SMG, "Selection procedures for the choice of radio transmission technologies of the UMTS," Tech. Rep. ETR/SMG 30.03, v.3.0.0, ETSI, Valbonne, France, 1997.

[2] M. Wahlqvist et al., "Description of Telias OFDM based proposal (working document in the OFDM concept group)," Tech. Rep. Tdoc 180/97, ETSI STC SMG2 meeting no 22, Bad Aibling, Germany, May 1997.

[3] J. A. C. Bingham, "Multicarrier modulation for data transmission: An idea whose time has come," IEEE Commun. Mag., vol. 28, pp. 5-14, May 1990.

[4] J.-J. van de Beek, M. Sandell, and P.-O. Börjesson, "ML estimation of timing and frequency offset in OFDM systems," IEEE Trans. Signal Proc., vol. 45, pp. 1800-1805, July 1997.

[5] M. Sandell, J.-J. van de Beek, and P. O. Börjesson, "Timing and frequency synchronization in OFDM systems using

\begin{tabular}{|l|l|}
\hline SNR $=10 \mathrm{~dB}$ & \\
\hline$\sqrt{E\left\{|\theta-\hat{\theta}|^{2}\right\}}$ & 6.41 samples \\
\hline $\mid \theta-\hat{\theta}>20$ samples & $0.98 \%$ \\
\hline $\mid \theta-\hat{\theta}>30$ samples & $0.08 \%$ \\
\hline $\mid \theta-\hat{\theta}>40$ samples & $0.01 \%$ \\
\hline$\sqrt{E\left\{|\varepsilon-\widehat{\varepsilon}|^{2}\right\}}$ & $1.17 \%$ \\
\hline$|\varepsilon-\hat{\varepsilon}|>1 \%$ & $35.22 \%$ \\
\hline$|\varepsilon-\hat{\varepsilon}|>2 \%$ & $8.32 \%$ \\
\hline$|\varepsilon-\hat{\varepsilon}|>3 \%$ & $1.96 \%$ \\
\hline
\end{tabular}

Table 2. Performance of the time and frequency offset estimates

the cyclic prefix," in Intern. Symp. Synch., (Essen, Germany), pp. 16-19, Dec. 1995.

[6] M. Mouly and M.-B. Pautet, The GSM System for Mobile Communications. Published by the authors, 1992.

[7] M. Moeneclaey, "The effect of synchronization errors on the performance of orthogonal frequency-division multiplexed (ofdm) systems," in Proceedings of the COST 254 workshop, (Toulouse, France), July 1997.

[8] P. Moose, "A technique for orthogonal frequency-division multiplexing frequency offset correction," IEEE Trans. Commun., vol. 42, pp. 2908-2914, Oct. 1994.

[9] L. Wei and C. Schlegel, "Synchronization requirements for multi-user OFDM on satellite mobile and two-path Rayleigh-fading channels," IEEE Trans. Commun., vol. 43, pp. 887-895, Feb/Mar/A pr 1995.

[10] T. Pollet, P. Spruyt, and M. Moeneclaey, "The BER performance of OFDM systems using non-synchronized sampling," in Proc. Globecom, vol. 1, (San Francisco, CA), pp. 253-257, Nov. 1994.

[11] T. Pollet and M. Moeneclaey, "Synchronizability of OFDM signals," in Proc. Globecom, vol. 3, (Singapore), pp. 20542058, Nov. 1995.

[12] J.- J. van de Beek et al., "Tracking of time and frequency offset in an OFDM-based system for UMTS," IEEE Transactions of Vehicular Technology, 1998. Submitted to.

[13] O. Edfors, M. Sandell, J.-J. van de Beek, S. K. Wilson, and P.-O. Börjesson, "OFDM channel estimation by singular value decomposition," IEEE Trans. Commun., 1997. Accepted for publication. 